Bull. Korean Math. Soc. 52 (2015), No. 1, pp. 35-43

http://dx.doi.org/10.4134/BKMS.2015.52.1.035

\title{
A NEW MEAN VALUE RELATED TO D. H. LEHMER'S PROBLEM AND KLOOSTERMAN SUMS
}

\author{
Di Han And Wenpeng Zhang
}

\begin{abstract}
Let $q>1$ be an odd integer and $c$ be a fixed integer with $(c, q)=1$. For each integer $a$ with $1 \leq a \leq q-1$, it is clear that there exists one and only one $b$ with $0 \leq b \leq q-1$ such that $a b \equiv c(\bmod q)$. Let $N(c, q)$ denote the number of all solutions of the congruence equation $a b \equiv c(\bmod q)$ for $1 \leq a, b \leq q-1$ in which $a$ and $\bar{b}$ are of opposite parity, where $\bar{b}$ is defined by the congruence equation $b \bar{b} \equiv 1(\bmod q)$. The main purpose of this paper is using the mean value theorem of Dirichlet $L$ functions to study the mean value properties of a summation involving $\left(N(c, q)-\frac{1}{2} \phi(q)\right)$ and Kloosterman sums, and give a sharper asymptotic formula for it.
\end{abstract}

\section{Introduction}

Let $p$ be an odd prime and $c$ be a fixed integer with $(c, p)=1$. For each integer $a$ with $1 \leq a \leq p-1$, it is clear that there exists one and only one $b$ with $0 \leq b \leq p-1$ such that $a b \equiv c(\bmod p)$. Let $M(c, p)$ denote the number of cases in which $a$ and $b$ are of opposite parity. In reference [4], D. H. Lehmer asked to study $M(1, p)$ or at least to say something nontrivial about it. It is known that $M(1, p) \equiv 2$ or $0(\bmod 4)$ when $p \equiv \pm 1(\bmod 4)$. For general odd number $q \geq 3$, Wenpeng Zhang [6] studied the asymptotic properties of $M(1, q)$, and obtained a sharp asymptotic formula:

$$
M(1, q)=\frac{1}{2} \phi(q)+O\left(q^{\frac{1}{2}} d^{2}(q) \ln ^{2} q\right),
$$

where $\phi(q)$ denotes the Euler function, and $d(q)$ is the number of divisors of $q$.

Recently, Wenpeng Zhang [9] considered the following problem: Let $q$ be an odd integer, $c$ be any integer with $(c, q)=1, N(c, q)$ denote the number of pairs of integers $a, b$ with $a b \equiv c(\bmod q)$ for $1 \leq a, b \leq q-1$ in which $a$ and

Received June 27, 2012

2010 Mathematics Subject Classification. Primary 11L40, 11F20.

Key words and phrases. D. H. Lehmer's problem, error term, Kloosterman sums, hybrid mean value, asymptotic formula.

This work is supported by the N.S.F. (11371291, 11471258) of P. R. China. 
$\bar{b}$ are of opposite parity, and

$$
E(c, q)=N(c, q)-\frac{1}{2} \phi(q) .
$$

Then he studied the computational problem of the mean value

$$
\sum_{\substack{c=1 \\(c, q)=1}}^{q} R_{q}(c+1) E(c, q),
$$

where $R_{q}(c)$ denotes the Ramanujan's sum, defined as (see Theorem 8.6 of [1]):

$$
R_{q}(c)=\sum_{\substack{k=1 \\(k, q)=1}}^{q} e^{\frac{2 \pi i k c}{q}}=\sum_{d \mid(c, q)} d \mu(q / d)
$$

where $\mu(n)$ is the famous Möbius function. Using the elementary and analytic methods, Wenpeng Zhang [9] proved the following conclusion:

Let $q \geq 3$ be an odd square-full number (that is, for any prime $p, p \mid q$ if and only if $\left.p^{2} \mid q\right)$. Then we have the identity

$$
\sum_{\substack{c=1 \\(c, q)=1}}^{q} R_{q}(c+1) E(c, q)=\frac{1}{2} \phi^{2}(q) \prod_{p \mid q}\left(1+\frac{1}{p}\right)
$$

where $\prod_{p \mid q}$ denotes the product over all prime divisors of $q$.

In this paper, we consider the asymptotic properties of the hybrid mean value related to $\mathrm{D}$. H. Lehmer's problem and Kloosterman sums:

$$
\sum_{m=1}^{q} \sum_{n=1}^{q} K(m u, m ; q) K(n u, n ; q) \cdot E\left(m^{2} \bar{n}^{2}, q\right),
$$

where $K(m, n ; q)=\sum_{a=1}^{\prime q} e\left(\frac{m a+n \bar{a}}{q}\right)$ denotes Kloosterman sums, $e(y)=e^{2 \pi i y}$ and $\bar{a}$ denotes the positive integer solution of the congruence equation $x a \equiv$ $1 \bmod q$.

About the asymptotic properties of (1), it seems that none had studied it yet, at least we have not seen any related result before. In this paper, we shall use the analytic methods and the estimation of Dirichlet character of polynomials to study this problem, and give an interesting asymptotic formula for it. That is, we shall prove the following conclusions:

Theorem 1. Let $p$ be an odd prime. Then for any integer $u$ with $(u, p)=1$, we have the asymptotic formula

$$
\sum_{m=1}^{p-1} \sum_{n=1}^{p-1} K(m u, m ; p) K(n u, n ; p) \cdot E\left(m^{2} \bar{n}^{2}, p\right)=-p^{3}+O\left(p^{\frac{5}{2}} \cdot \ln ^{2} p\right) .
$$


Theorem 2. Let $p$ be an odd prime with $p \equiv 3 \bmod 4$. Then for any nonprincipal even character $\chi \bmod p$, we have the identity

$$
\sum_{m=1}^{p-1}\left|\sum_{a=1}^{p-1} \chi(m a+\bar{a})\right|^{2} \cdot E\left(m^{2}, p\right)=-p \cdot\left(20-16\left(\frac{2}{p}\right)\right) \cdot h_{p}^{2},
$$

where $h_{p}$ denotes the class number of the quadratic field $\mathbf{Q}(\sqrt{-p})$, and $\left(\frac{*}{p}\right)$ denotes the Legendre's symbol.

It is very interesting that from our Theorem 2 we can give a new expression for the class number of the quadratic field $\mathbf{Q}(\sqrt{-p})$, i.e.,

$$
h_{p}^{2}=\frac{-1}{p\left(20-16\left(\frac{2}{p}\right)\right)} \sum_{m=1}^{p-1}\left|\sum_{a=1}^{p-1} \chi(m a+\bar{a})\right|^{2} \cdot E\left(m^{2}, p\right)
$$

holds for any non-principal character $\chi \bmod p$ with $p \equiv 3 \bmod 4$.

\section{Several lemmas}

In this section, we shall give several lemmas, which are necessary in the proof of our theorems. First we have the following:

Lemma 1. Let $p>1$ be an odd prime. Then for any integer $c$ with $(c, p)=1$, we have the identity

$$
E(c, p)=-\frac{2}{\pi^{2}} \cdot \frac{p}{p-1} \cdot \sum_{\substack{\chi \bmod p \\ \chi(-1)=-1}} \bar{\chi}(c) \cdot|1-2 \chi(2)|^{2} \cdot|L(1, \chi)|^{2},
$$

where $\sum_{\substack{\chi \bmod p \\ \chi(-1)=-1}}$ denotes the summation over all odd characters $\chi \bmod p$.

Proof. From the orthogonality relation for character sums $\bmod p$ and the definition of $N(c, p)$, we have

$$
\begin{aligned}
N(c, p) & =\frac{1}{2} \sum_{\substack{a=1 \\
a b \equiv c \bmod p}}^{p} \sum_{b=1}^{p}\left(1-(-1)^{a+\bar{b}}\right) \\
& =\frac{1}{2} \phi(p)-\frac{1}{2} \sum_{\substack{a=1 \\
a b \equiv c \bmod p}}^{p}(-1)^{a+\bar{b}} \\
& =\frac{1}{2} \phi(p)-\frac{1}{2 \phi(p)} \sum_{\chi \bmod p} \bar{\chi}(c)\left(\sum_{a=1}^{p}(-1)^{a} \chi(a)\right)\left(\sum_{b=1}^{p}(-1)^{\bar{b}} \chi(b)\right) \\
& =\frac{1}{2} \phi(p)-\frac{1}{2 \phi(p)} \sum_{\chi \bmod p} \bar{\chi}(c)\left(\sum_{a=1}^{p}(-1)^{a} \chi(a)\right)\left(\sum_{b=1}^{p}(-1)^{b} \chi(\bar{b})\right)
\end{aligned}
$$




$$
\begin{aligned}
& =\frac{1}{2} \phi(p)-\frac{1}{2 \phi(p)} \sum_{\chi \bmod p} \bar{\chi}(c)\left(\sum_{a=1}^{p}(-1)^{a} \chi(a)\right) \overline{\left(\sum_{b=1}^{p}(-1)^{b} \chi(b)\right)} \\
& =\frac{1}{2} \phi(p)-\frac{1}{2 \phi(p)} \sum_{\chi \bmod p} \bar{\chi}(c)\left|\sum_{a=1}^{p}(-1)^{a} \chi(a)\right|^{2}
\end{aligned}
$$

It is clear that $\sum_{a=1}^{p}(-1)^{a} \chi(a)=0$, if $\chi(-1)=1$. And $\sum_{a=1}^{p}(-1)^{a} \chi(a)=$ $2 \chi(2) \sum_{a=1}^{\frac{p-1}{2}} \chi(a)$, if $\chi(-1)=-1$. For any odd character $\chi \bmod p$, note that the identities (see Theorems 12.11 and 12.20 of [1])

$$
\left|\frac{1}{p} \sum_{b=1}^{p-1} b \chi(b)\right|=\frac{\sqrt{p}}{\pi} \cdot|L(1, \bar{\chi})|
$$

and (see reference [3])

$$
(1-2 \chi(2)) \sum_{a=1}^{p} a \chi(a)=\chi(2) p \sum_{a=1}^{\frac{p-1}{2}} \chi(a) .
$$

From (2) and the definition of $E(c, p)$ we may immediately deduce the identity

$$
E(c, p)=-\frac{2}{\pi^{2}} \cdot \frac{p}{p-1} \cdot \sum_{\substack{\chi \bmod p \\ \chi(-1)=-1}} \bar{\chi}(c) \cdot|1-2 \chi(2)|^{2} \cdot|L(1, \chi)|^{2} .
$$

This proves Lemma 1.

Lemma 2. Let $p$ be an odd prime, $\chi$ be any non-principal even character $\bmod p$. Then for any integer $m$ with $(m, p)=1$, we have the identity

$$
\left|\sum_{a=1}^{p-1} \chi(m a+\bar{a})\right|^{2}=2 p+\left(\frac{m}{p}\right) \sum_{a=1}^{p-1} \chi(a) \sum_{b=1}^{p-1}\left(\frac{b(b-1)\left(a^{2} b-1\right)}{p}\right)
$$

where $\left(\frac{*}{p}\right)$ denotes the Legendre's symbol.

Proof. Let $a m+\bar{a}=u$. Then from the definition of $\bar{a}$ and the properties of congruence $\bmod p$ we know that for any $(m, p)=1$, we have

$$
\begin{aligned}
\sum_{a=1}^{p-1} \chi(m a+\bar{a}) & =\sum_{u=1}^{p-1} \chi(u) \sum_{\substack{a=1 \\
a m+\bar{a} \equiv u \bmod p}}^{p-1} 1=\sum_{u=1}^{p-1} \chi(u) \sum_{\substack{a=1 \\
a^{2} m^{2}-a m u+m \equiv 0 \bmod p}}^{p-1} 1 \\
& =\sum_{u=1}^{p-1} \chi(u) \quad \sum_{\substack{a=0 \\
(3)}}^{p-1} 1=\sum_{\substack{a=1 \\
(2 a m-u)^{2} \equiv u^{2}-4 m \bmod p}}^{p-1} \chi(u) \sum_{\substack{a=0 \\
a^{2} \equiv u^{2}-4 m \bmod p}}^{p-1} 1 .
\end{aligned}
$$


Note that for any fixed integer $u^{2}-4 m$, the number of the solutions of the congruent equation $x^{2} \equiv u^{2}-m \bmod p$ are $1+\left(\frac{u^{2}-4 m}{p}\right)$, so from (3) we have

(4) $\sum_{a=1}^{p-1} \chi(m a+\bar{a})=\sum_{u=1}^{p-1} \chi(u)\left(1+\left(\frac{u^{2}-4 m}{p}\right)\right)=\chi(2) \sum_{u=1}^{p-1} \chi(u)\left(\frac{u^{2}-m}{p}\right)$.

Now from (4) and the properties of reduced residue system $\bmod p$ we have

$$
\begin{aligned}
\left|\sum_{a=1}^{p-1} \chi(m a+\bar{a})\right|^{2}= & \left|\sum_{u=1}^{p-1} \chi(u)\left(\frac{u^{2}-m}{p}\right)\right|^{2} \\
= & \sum_{a=1}^{p-1} \sum_{b=1}^{p-1} \chi(a \bar{b})\left(\frac{a^{2}-m}{p}\right)\left(\frac{b^{2}-m}{p}\right) \\
= & \sum_{a=1}^{p-1} \chi(a) \sum_{b=1}^{p-1}\left(\frac{a^{2} b^{2}-m}{p}\right)\left(\frac{b^{2}-m}{p}\right) \\
= & \sum_{a=1}^{p-1} \chi(a) \sum_{b=1}^{p-1}\left(1+\left(\frac{b}{p}\right)\right)\left(\frac{a^{2} b-m}{p}\right)\left(\frac{b-m}{p}\right) \\
= & \sum_{a=1}^{p-1} \chi(a) \sum_{b=1}^{p-1}\left(\frac{\left(a^{2} b-1\right)(b-1)}{p}\right) \\
& +\left(\frac{m}{p}\right) \sum_{a=1}^{p-1} \chi(a) \sum_{b=1}^{p-1}\left(\frac{\left(a^{2} b-1\right) b(b-1)}{p}\right) .
\end{aligned}
$$

Note that $\chi(-1)=1$. From the properties of the complete residue system $\bmod p$ we also have

$$
\begin{aligned}
\sum_{a=1}^{p-1} \chi(a) \sum_{b=1}^{p-1}\left(\frac{\left(a^{2} b-1\right)(b-1)}{p}\right) & =\sum_{a=1}^{p-1} \chi(a) \sum_{b=0}^{p-1}\left(\frac{\left(2 a^{2} b-a^{2}-1\right)^{2}-\left(a^{2}-1\right)^{2}}{p}\right) \\
& =\sum_{a=1}^{p-1} \chi(a) \sum_{b=0}^{p-1}\left(\frac{b^{2}-\left(a^{2}-1\right)^{2}}{p}\right)
\end{aligned}
$$

and

$$
\sum_{a=1}^{p}\left(\frac{a^{2}+n}{p}\right)= \begin{cases}-1, & \text { if }(n, p)=1 \\ p-1, & \text { if }(n, p)=p .\end{cases}
$$

Combining (6) and (7) we can deduce the identity

$$
\sum_{a=1}^{p-1} \chi(a) \sum_{b=1}^{p-1}\left(\frac{\left(a^{2} b-1\right)(b-1)}{p}\right)=2(p-1)-\sum_{a=2}^{p-2} \chi(a)=2 p .
$$

Now Lemma 2 follows from (5) and (8). 
Lemma 3. For any odd prime $p$, we have the estimate

$$
\left.\sum_{a=1}^{p-1}\left|\sum_{\substack{\chi \bmod p \\ \chi(-1)=-1}} \chi(a)\right| L(1, \chi)\right|^{2} \mid=O(p \cdot \ln p) .
$$

Proof. See Lemma 1 of [8].

\section{Proof of the theorems}

In this section, we shall complete the proof of our theorems. First we prove Theorem 1. From the properties of the classical Gauss sums we know that $|\tau(\chi)|=\sqrt{p}$, if $\chi$ is not a principal character $\bmod p$. And $|\tau(\chi)|=1$, if $\chi=\chi_{0}$ is the principal character mod $p$. If $\chi(-1)=-1$, then $\chi^{2}$ must be an even character $\bmod p$. So from Lemma 1 and the definition of Gauss sums we have

$$
\text { (9) } \begin{aligned}
& \sum_{m=1}^{p-1} \sum_{n=1}^{p-1} K(m u, m ; p) K(n u, n ; p) \cdot E\left(m^{2} \bar{n}^{2}, p\right) \\
= & \frac{2}{\pi^{2}} \frac{-p}{p-1} \sum_{\substack{\chi \bmod p \\
\chi(-1)=-1}}\left|\sum_{m=1}^{p-1} \bar{\chi}^{2}(m) \sum_{a=1}^{p-1} e\left(\frac{m u a+m \bar{a}}{p}\right)\right|^{2} \cdot|1-2 \chi(2)|^{2} \cdot|L(1, \chi)|^{2} \\
= & \frac{2}{\pi^{2}} \frac{-p}{p-1} \sum_{\substack{\chi \bmod p \\
\chi(-1)=-1}}\left|\sum_{a=1}^{p-1} \sum_{m=1}^{p-1} \bar{\chi}^{2}(m) e\left(\frac{m(u a+\bar{a})}{p}\right)\right|^{2} \cdot(5-4 \chi(2)) \cdot|L(1, \chi)|^{2} \\
= & \frac{2}{\pi^{2}} \frac{-p}{p-1} \sum_{\substack{\chi \bmod p \\
\chi(-1)=-1}}\left|\tau\left(\bar{\chi}^{2}\right)\right|^{2}\left|\sum_{a=1}^{p-1} \chi^{2}(u a+\bar{a})\right|^{2} \cdot(5-4 \chi(2)) \cdot|L(1, \chi)|^{2} \\
= & \frac{2}{\pi^{2}} \frac{-p^{2}}{p-1} \sum_{\substack{\chi \bmod p \\
\chi(-1)=-1 \\
\chi^{2} \neq \chi_{0}}}\left|\sum_{a=1}^{p-1} \chi^{2}(u a+\bar{a})\right|^{2} \cdot(5-4 \chi(2)) \cdot|L(1, \chi)|^{2} \\
& -\frac{1}{\pi^{2}} \frac{p}{p-1}\left(1-\left(\frac{-1}{p}\right)\right)\left|\sum_{a=1}^{p-1} \chi_{0}(u a+\bar{a})\right|^{2}\left(5-4\left(\frac{2}{p}\right)\right)\left|L\left(1, \chi_{2}\right)\right|^{2},
\end{aligned}
$$

where we have used the identity $\sum_{m=1}^{p-1} \chi(m) e\left(\frac{m a}{p}\right)=\bar{\chi}(a) \sum_{m=1}^{p-1} e\left(\frac{m}{p}\right)=$ $\bar{\chi}(a) \tau(\chi)$. 
Note that the number of the solutions of the congruent equation $a^{2} u+1 \equiv$ $0 \bmod p$ is $1+\left(\frac{-u}{p}\right), L\left(1, \chi_{2}\right)=\pi h_{p} / \sqrt{p}$, so we have

$$
\left|\sum_{a=1}^{p-1} \chi_{0}(u a+\bar{a})\right|^{2}\left|L\left(1, \chi_{2}\right)\right|^{2}=\frac{1}{p}\left(p-2-\left(\frac{-u}{p}\right)\right)^{2} \cdot \pi^{2} \cdot h_{p}^{2},
$$

where $h_{p}$ denotes the class number of the quadratic field $\mathbf{Q}(\sqrt{-p})$.

For any integer $a$, from the classical estimate of Weil (see [2]) we have the estimate

$$
\left|\sum_{b=1}^{p-1}\left(\frac{b(b-1)(a b-1)}{p}\right)\right| \ll \sqrt{p} \cdot \ln p
$$

and the identities (see [5], [7] or [9])

$$
\begin{aligned}
& \sum_{\substack{\chi \bmod p \\
\chi(-1)=-1}}|L(1, \chi)|^{2}=\frac{\pi^{2}}{12} \cdot \frac{(p-1)^{2}(p-2)}{p^{2}}, \\
& \sum_{\substack{\chi \bmod p \\
\chi(-1)=-1}} \chi(2)|L(1, \chi)|^{2}=\frac{\pi^{2}}{24} \frac{(p-1)^{2}(p-5)}{p^{2}} .
\end{aligned}
$$

If $p \equiv 1 \bmod 4$, note that there does not exist character $\chi \bmod p$ with $\chi(-1)=-1$ such that $\chi^{2}=\chi_{0}$. So applying (11), (12), (13), Lemma 1 and Lemma 3 we have

(14)

$$
\begin{aligned}
& \sum_{\substack{\chi \bmod p \\
\chi(-1)=-1 \\
\chi^{2} \neq \chi_{0}}}\left|\sum_{a=1}^{p-1} \chi^{2}(u a+\bar{a})\right|^{2} \cdot(5-4 \chi(2)) \cdot|L(1, \chi)|^{2} \\
&= \sum_{\substack{\chi \bmod p \\
\chi(-1)=-1}}\left|\sum_{a=1}^{p-1} \chi^{2}(u a+\bar{a})\right|^{2} \cdot(5-4 \chi(2)) \cdot|L(1, \chi)|^{2} \\
&= 2 p \sum_{\substack{\chi \bmod p \\
\chi(-1)=-1}}(5-4 \chi(2)) \cdot|L(1, \chi)|^{2} \\
&+\sum_{\substack{\chi \bmod p \\
\chi(-1)=-1}}\left(\frac{u}{p}\right) \sum_{a=1}^{p-1} \chi^{2}(a) \sum_{b=1}^{p-1}\left(\frac{b(b-1)\left(a^{2} b-1\right)}{p}\right) \cdot(5-4 \chi(2)) \cdot|L(1, \chi)|^{2}
\end{aligned}
$$




$$
\begin{aligned}
= & \left(\frac{u}{p}\right) \sum_{a=1}^{p-1}\left(1+\left(\frac{a}{p}\right)\right) \sum_{b=1}^{p-1}\left(\frac{b(b-1)(a b-1)}{p}\right) \sum_{\substack{\chi \bmod p \\
\chi(-1)=-1}}(5 \chi(a)-4 \chi(2 a)) \cdot|L(1, \chi)|^{2} \\
& +\frac{5 \pi^{2}}{6} \cdot \frac{(p-1)^{2}(p-2)}{p}-\frac{2 \pi^{2}}{6} \cdot \frac{(p-1)^{2}(p-5)}{p} \\
= & \left.\frac{\pi^{2}}{2} \cdot(p-1)^{2}+O\left|\sum_{a=1}^{p-1}\right| \sum_{b=1}^{p-1}\left(\frac{b(b-1)(a b-1)}{p}\right)|\cdot| \sum_{\chi \bmod p} \chi(a)|L(1, \chi)|^{2} \mid\right) \\
& \left.+O\left|\sum_{a=1}^{p-1}\right| \sum_{b=1}^{p-1}\left(\frac{b(b-1)(a b-1)}{p}\right)|\cdot| \sum_{\substack{\chi \bmod p \\
\chi(-1)=-1}} \chi(2 a)|L(1, \chi)|^{2} \mid\right) \\
= & \left.\frac{\pi^{2}}{2} \cdot p^{2}+O\left(p^{\frac{3}{2}} \cdot \ln ^{2} p\right) \cdot \mid\right)
\end{aligned}
$$

Now combining (9) and (14) we may immediately deduce the asymptotic formula

(15) $\sum_{m=1}^{p-1} \sum_{n=1}^{p-1} K(m u, m ; p) K(n u, n ; p) \cdot E\left(m^{2} \bar{n}^{2}, p\right)=-p^{3}+O\left(p^{\frac{5}{2}} \cdot \ln ^{2} p\right)$.

If $p \equiv-1 \bmod 4$, note that $h_{p}=O(\sqrt{p} \cdot \ln p)$, from $(9),(10)$ and the method of proving (14) we can also deduce that the asymptotic formula (15) holds. This completes the proof of Theorem 1 .

Now we prove Theorem 2. For any non-principal even character $\chi_{1} \bmod p$, from Lemma 1 and Lemma 2 we have

$$
\begin{aligned}
& \sum_{m=1}^{p-1}\left|\sum_{a=1}^{p-1} \chi_{1}(m a+\bar{a})\right|^{2} \cdot E\left(m^{2}, p\right) \\
= & \frac{2}{\pi^{2}} \cdot \frac{-p}{p-1} \sum_{\substack{\chi \bmod p \\
\chi(-1)=-1}} \sum_{m=1}^{p-1} \bar{\chi}^{2}(m)\left|\sum_{a=1}^{p-1} \chi_{1}(m a+\bar{a})\right|^{2} \cdot|1-2 \chi(2)|^{2} \cdot|L(1, \chi)|^{2} \\
= & \frac{4}{\pi^{2}} \cdot \frac{-p^{2}}{p-1} \sum_{\substack{\chi \bmod p \\
\chi(-1)=-1}}\left(\sum_{m=1}^{p-1} \bar{\chi}^{2}(m)\right) \cdot(5-4 \chi(2)) \cdot|L(1, \chi)|^{2} \\
& -\frac{2}{\pi^{2}} \cdot \frac{p}{p-1} \sum_{\substack{\chi \bmod p \\
\chi(-1)=-1}}\left(\sum_{m=1}^{p-1} \bar{\chi}^{2}(m)\left(\frac{m}{p}\right)\right) \cdot(5-4 \chi(2)) \cdot|L(1, \chi)|^{2}
\end{aligned}
$$




$$
\times\left(\sum_{a=1}^{p-1} \chi_{1}(a) \sum_{b=1}^{p-1}\left(\frac{b(b-1)\left(a^{2} b-1\right)}{p}\right)\right) .
$$

Note that if $p \equiv 3 \bmod 4$, then for any $\chi \bmod p$ with $\chi(-1)=-1$, we have

$$
\sum_{m=1}^{p-1} \bar{\chi}^{2}(m)\left(\frac{m}{p}\right)=0 .
$$

And if $\chi=\left(\frac{*}{p}\right)$, then $\sum_{m=1}^{p-1} \bar{\chi}^{2}(m)=p-1$; If $\chi \neq\left(\frac{*}{p}\right)$, then $\sum_{m=1}^{p-1} \bar{\chi}^{2}(m)=0$. So from (16) and $L\left(1, \chi_{2}\right)=\pi h_{p} / \sqrt{p}$ we may immediately deduce the identity

$$
\begin{aligned}
& \sum_{m=1}^{p-1}\left|\sum_{a=1}^{p-1} \chi_{1}(m a+\bar{a})\right|^{2} \cdot E\left(m^{2}, p\right) \\
= & -4 p\left(5-4\left(\frac{2}{p}\right)\right) \cdot h_{p}^{2}=-p \cdot\left(20-16\left(\frac{2}{p}\right)\right) \cdot h_{p}^{2} .
\end{aligned}
$$

This completes the proof of Theorem 2 .

\section{References}

[1] T. M. Apostol, Introduction to Analytic Number Theory, Springer-Verlag, New York, 1976 .

[2] D. A. Burgess, On Dirichlet characters of polynomials, Proc. London Math. Soc. 13 (1963), 537-548.

[3] T. Funakura, On Kronecker's limit formula for Dirichlet series with periodic coefficients, Acta Arith. 55 (1990), no. 1, 59-73.

[4] R. K. Guy, Unsolved Problems in Number Theory, Second Edition, Springer-Verlag, New York, 1994

[5] H. Walum, An exact formula for an average of L-series, Illinois J. Math. 26 (1982), no. $1,1-3$.

[6] W. Zhang, On a problem of D. H. Lehmer and its generalization, Compositio Math. 86 (1993), no. 3, 307-316.

[7] A note on the mean square value of the Dedekind sums, Acta Math. Hungar. 86 (2000), no. $4,275-289$.

[8] $\longrightarrow$ Moments of generalized quadratic Gauss sums weighted by L-functions, J. Number Theory 92 (2002), no. 2, 304-314.

[9] _ A mean value related to D. H. Lehmer's problem and the Ramanujan's sum, Glasg. Math. J. 54 (2012), no. 1, 155-162.

Di HAN

School of Mathematics

NORTHWEST UNIVERSITY

Xi'An, ShaAnXi, P. R. China

E-mail address: handi515@163.com

WENPENG ZHANG

SChOOL OF Mathematics

NORTHWEST UNIVERSITY

Xi'An, ShaAnXi, P. R. China

E-mail address: wpzhang@nwu.edu.cn 